\title{
High Power Thermoelectrically Cooled and Uncooled Quantum Cascade Lasers With Optimized Reflectivity Facet Coatings
}

\section{Citation}

Maulini, Richard, Arkadiy Lyakh, Alexei Tsekoun, Rowel Go, Christian Pflügl, Laurent Diehl, Federico Capasso, and C. Kumar N. Patel. 2009. High power thermoelectrically cooled and uncooled quantum cascade lasers with optimized reflectivity facet coatings. Applied Physics Letters (95)15: 151112.

\section{Published Version}

http://dx.doi.org/10.1063/1.3246799

\section{Permanent link}

http://nrs.harvard.edu/urn-3:HUL.InstRepos:3426316

\section{Terms of Use}

This article was downloaded from Harvard University's DASH repository, and is made available under the terms and conditions applicable to Other Posted Material, as set forth at http:// nrs.harvard.edu/urn-3:HUL.InstRepos:dash.current.terms-of-use\#LAA

\section{Share Your Story}

The Harvard community has made this article openly available.

Please share how this access benefits you. Submit a story.

Accessibility 


\title{
High power thermoelectrically cooled and uncooled quantum cascade lasers with optimized reflectivity facet coatings
}

\author{
Richard Maulini, ${ }^{1, \text { a) }}$ Arkadiy Lyakh, ${ }^{1}$ Alexei Tsekoun, ${ }^{1}$ Rowel Go, ${ }^{1}$ Christian Pflügl, ${ }^{2}$ \\ Laurent Diehl, ${ }^{2}$ Federico Capasso, ${ }^{2}$ and C. Kumar N. Patel ${ }^{1,3}$ \\ ${ }_{1}^{1}$ Pranalytica, Inc., 1101 Colorado Avenue, Santa Monica, California 90401, USA \\ ${ }^{2}$ School of Engineering and Applied Sciences, Harvard University, Cambridge, Massachusetts 02138, USA \\ ${ }^{3}$ Department of Physics and Astronomy, University of California, Los Angeles, California 91748, \\ USA
}

(Received 31 July 2009; accepted 21 September 2009; published online 15 October 2009)

\begin{abstract}
We present a method of preserving the device wall-plug efficiency by adjusting mirror losses with facet coatings for longer cavity quantum cascade lasers. An experimental study of output power and wall-plug efficiency as functions of mirror losses was performed by varying the front facet coating reflectivity with a high-reflectivity-coated rear facet. The use of optimized reflectivity coatings on 7-mm-long chips resulted in continuous-wave output power of $2.9 \mathrm{~W}$ at $293 \mathrm{~K}$ for thermoelectrically cooled devices mounted on AlN submounts and average and continuous-wave output power in excess of $1 \mathrm{~W}$ for uncooled devices emitting at $4.6 \mu \mathrm{m}$. (C) 2009 American Institute of Physics. [doi:10.1063/1.3246799]
\end{abstract}

Midwave infrared (MWIR) quantum cascade lasers (QCLs) with watt-level continuous-wave (cw) output power at room temperature have recently been demonstrated. ${ }^{1-4}$ These lasers are useful for applications such as protecting aircraft from shoulder-fired missiles, free-space optical communications, infrared illuminators for night-vision applications, and chemical sensing. All these applications would benefit from higher power, improved reliability, and lower power consumption.

The large design flexibility of QCLs allows one to scale up their output power in several ways: increase the number of active region stages, increase the active region sheet carrier density per stage, and increase the chip dimensions, i.e., width and/or length. All these approaches have been demonstrated to result in higher peak power in pulsed mode, but most of them are of limited usefulness for $\mathrm{cw}$ devices because of active region self-heating. An increase in the number of stages or the sheet carrier density result in a higher generated heat per unit area in the active region and, thus, in a higher temperature rise. An increase in the active region width results in an increase in thermal resistance and thus leads to a temperature rise. Moreover, unless special measures are taken, wider waveguides result in lower beam quality.

The approach which consists of increasing the laser cavity length does not suffer, on the other hand, from higher active region temperature or lower beam quality. In this case, however, the mirror losses need to be optimized to maximize the wall-plug efficiency (WPE). Another advantage of this approach is that high power can be obtained from narrow devices, which are less subject to self-heating and are thus less sensitive to limitations such as the thermal conductivity of the submount. Up to now, cw output power in excess of $2 \mathrm{~W}$ has been achieved only with devices mounted on diamond submounts with indium. ${ }^{3,4}$ Diamond has the advantage of a very high thermal conductivity, but because of the large mismatch between its coefficient of thermal expansion

${ }^{a)}$ Electronic mail: rmaulini@pranalytica.com.
(CTE) and that of InP-based QCLs, it has to be used together with a soft solder material such as indium. In solder has been shown to be unsuitable for high-reliability operation of high power semiconductor lasers because of its migration at high temperatures and current densities. ${ }^{5}$ High-reliability operation requires the use of a hard solder and a CTE-matched submount to prevent failure due to mechanical stress. In this letter, we report close to $3 \mathrm{~W}$ of $\mathrm{cw}$ power from a chip mounted on AlN with AuSn. ${ }^{6}$ We also report more than $1 \mathrm{~W}$ of $\mathrm{cw}$ power from an uncooled QCL.

In the simple Rigrod analysis, i.e., assuming uniform gain saturation, ${ }^{7}$ the device WPE $\eta_{\mathrm{WPE}}$ at the roll-over current density $J_{\max }$ can be written as ${ }^{8}$

$$
\eta_{\mathrm{WPE}}=\frac{N h \nu}{e V_{\max }}\left[1-\frac{1}{J_{\max }}\left(J_{\mathrm{tr}}+\frac{\alpha_{w}+\alpha_{m}}{g \Gamma}\right)\right] \eta_{i} \frac{\alpha_{m}}{\alpha_{w}+\alpha_{m}},
$$

where $N$ is the number of stages, $h \nu$ is the photon energy, $V_{\max }$ is the voltage at $J_{\max }, J_{\text {tr }}$ is the transparency current density, $\alpha_{w}$ is the waveguide losses, $\alpha_{m}$ is the mirror losses, $g \Gamma$ is the differential modal gain, and $\eta_{i}$ is the internal quantum efficiency per stage. As discussed later in text, the WPE reaches its maximum for a length $L_{\mathrm{op}}$, which is typically equal to $\sim 3 \mathrm{~mm}$ for our $4.6 \mu \mathrm{m}$ QCLs with high-reflectivity (HR) coated rear facet and uncoated front facet. Since the WPE depends on $L$ only through the mirror losses $\alpha_{m}=$ $-1 /(2 L) \ln (R)$, where $R$ is the reflectivity of the output facet (assuming the other facet is HR coated), it can be maximized for a given cavity length by deposition of a facet coating that will produce the same mirror losses as those for the optimal length $L_{\text {opt }}$. However, as pointed out by Schindler, ${ }^{9}$ nonuniform gain saturation, due to nonuniform longitudinal intensity profile in the waveguide, has a detrimental effect on the WPE of long devices. This effect will be discussed later in the paper.

Experimentally, we reduced the facet reflectivity of our chips by depositing a single layer of $\mathrm{Al}_{2} \mathrm{O}_{3}$. The chips were based on our nonresonant extraction active region design, ${ }^{2}$ grown by molecular beam epitaxy, and processed as buried heterostructures (BHs) as described in Ref. 4. The refractive 


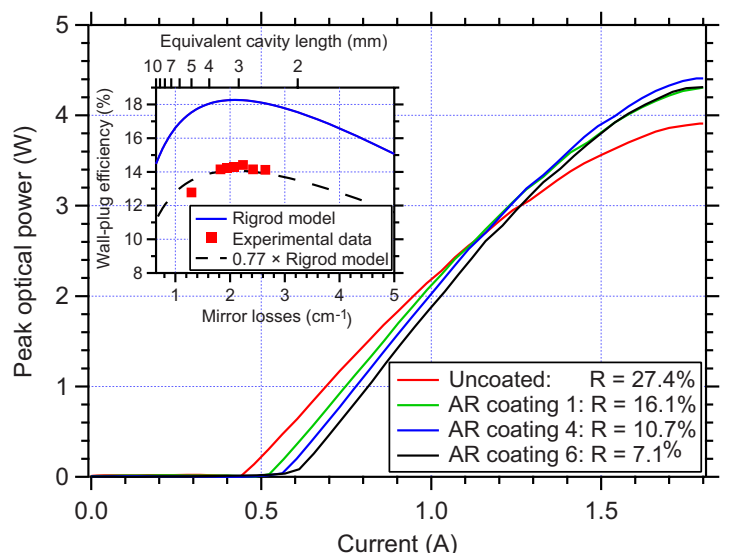

FIG. 1. (Color online) Peak output power as a function of injection current for a QCL with different AR coatings. The back facet of the device was HR coated. Inset: measured and predicted WPE as function of mirror losses for the same device.

index of alumina at the laser wavelength $(4.6 \mu \mathrm{m})$ is close to 1.6, which allows us to tailor the reflectivity between $27 \%$ (uncoated) and $\sim 1 \%$ (quarter-wave layer) by adjusting the layer thickness.

First, a HR coating was deposited on the back facet of a 5-mm-long, 11- $\mu \mathrm{m}$-wide sample mounted episide down on an AlN submount and a reference light-current-voltage (LIV) characteristic was measured. An antireflection (AR) coating was then deposited on its front facet and its thickness was varied from 350 to $500 \mathrm{~nm}$ in $30 \mathrm{~nm}$ increments by successive depositions, corresponding to a variation in facet reflectivity from $16 \%$ to $7 \%$ and a variation in mirror losses from 1.83 to $2.65 \mathrm{~cm}^{-1}$. LIV's were measured after each deposition. Four representative optical power versus current characteristics are displayed in Fig. 1. The maximum peak output power increased from $3.9 \mathrm{~W}$ without AR coating up to a maximum of $4.4 \mathrm{~W}$ for a facet reflectivity of $10.7 \%$, corresponding to an equivalent cavity length of $2.9 \mathrm{~mm}$, before decreasing again. This result agrees with our previous study of WPE versus cavity length for similar chips with HR/ uncoated facets, which resulted in an optimum length of $3 \mathrm{~mm}$.

By plotting the measured threshold current density as a function of mirror losses and the measured reciprocal slope efficiency at threshold as a function of reciprocal mirror losses, we were able to deduce the internal parameters of the laser. $^{8} \quad J_{\mathrm{tr}}=0.44 \pm 0.05 \mathrm{kA} / \mathrm{cm}^{2}, g \Gamma=4.7 \pm 0.3 \mathrm{~cm} / \mathrm{kA}, \quad \alpha_{w}$ $=0.5 \pm 0.1 \mathrm{~cm}^{-1}$, and $\eta_{i}=0.5 \pm 0.1$. This method is an interesting variation in the traditional method based on measurements of a set of chips of different lengths, whose precision is limited by chip to chip performance variations. Wittmann et al. ${ }^{10}$ described the transparency current in QCLs by introducing additional resonant waveguide losses $\alpha_{w, \text { res }}=g \Gamma J_{\text {tr }}$. In this equivalent formalism, we get $\alpha_{w, \text { res }}=2.1 \mathrm{~cm}^{-1}$. The total waveguide losses, in this formalism, $\alpha_{w}+\alpha_{w, \text { res }}=2.6 \mathrm{~cm}^{-1}$ are thus strongly dominated by resonant losses due to thermal population of the lower laser level.

The inset in Fig. 1 shows the measured WPE plotted along with the WPE predicted by Eq. (1) using the parameters given above. The measured WPE is significantly lower than predicted by the model because of the decrease in the slope efficiency between threshold and roll-over. Good agreement between model and measurements is obtained by multiplying the model data by 0.77 to take this effect into

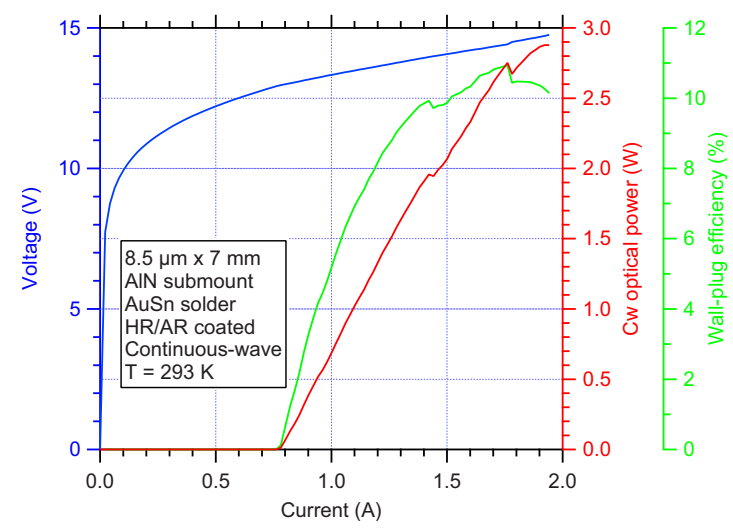

FIG. 2. (Color online) $\mathrm{cW}$ output power, voltage, and WPE as functions of the current for a 7-mm-long, thermoelectrically cooled, HR/AR coated QCL operating at room temperature.

account. Our model predicts an optimum cavity length of $L_{\text {opt }}=3.1 \mathrm{~mm}$, also in good agreement with the experimental data. The longest cavity length for which we can maximize the WPE using our single layer coating recipe is $\sim 10 \mathrm{~mm}$, for which the necessary facet reflectivity is $\sim 1 \%$.

We applied a similar combination of HR and AR coatings to 7-mm-long BH devices from another epiwafer based on the same active region and waveguide, but with 1.7 times higher active region doping. Despite the higher doping level, we chose an AR coating reflectivity of $4.9 \%$, corresponding to an equivalent cavity length of $3 \mathrm{~mm}$, based on the results reported above. The chips were mounted episide down on AlN carriers with AuSn solder.

For cw operation, chip-on-carrier assemblies were installed in butterfly-type packages equipped with a thermoelectric cooler (TEC) and collimating optics. The packages were then hermetically sealed under nitrogen atmosphere and mounted on a water-cooled heat sink. The voltage, output power, and WPE versus current characteristics of an 8.5- $\mu \mathrm{m}$-wide, 7-mm-long chip measured in $\mathrm{cw}$ operation at $293 \mathrm{~K}$ are shown in Fig. 2. The maximum output power, corrected for the lens collection efficiency, was $2.88 \mathrm{~W}$. Although we recently reported slightly higher $\mathrm{cw}$ power at room temperature $(\sim 3 \mathrm{~W})$ using wider, shorter chips mounted on diamond submounts with indium solder, ${ }^{4}$ the present result is significant because it was achieved with AlN submounts and AuSn solder, which have superior long-term reliability. The maximum WPE was $10.9 \%$, slightly lower than we have previously reported for shorter devices mounted on AlN (13\%). ${ }^{4}$ This difference is attributed to higher active region doping.

For high-duty-cycle pulsed operation, chip-on-carrier assemblies were installed into similar packages with collimating optics, but without TEC. The packages were then sealed and mounted on a passive metallic heat sink attached to the optical table. Heat dissipation did not rely on air or water cooling but only on conductive heat transfer through the metallic heatsink. This mode of operation of semiconductor lasers is usually referred to as uncooled operation. The lasers were operated in pulsed mode at a current density of $\sim 4 \mathrm{kA} / \mathrm{cm}^{2}$ with a pulse width of $200 \mathrm{~ns}$ and repetition rates varying between 0.5 and $4 \mathrm{MHz}$. Average output power as a function of duty cycle of two 7-mm-long devices of 8.5 and $5 \mu \mathrm{m}$ ridge widths is shown in the top part of Fig. 3. The average output power of the 8.5 - $\mu \mathrm{m}$-wide chip was observed 

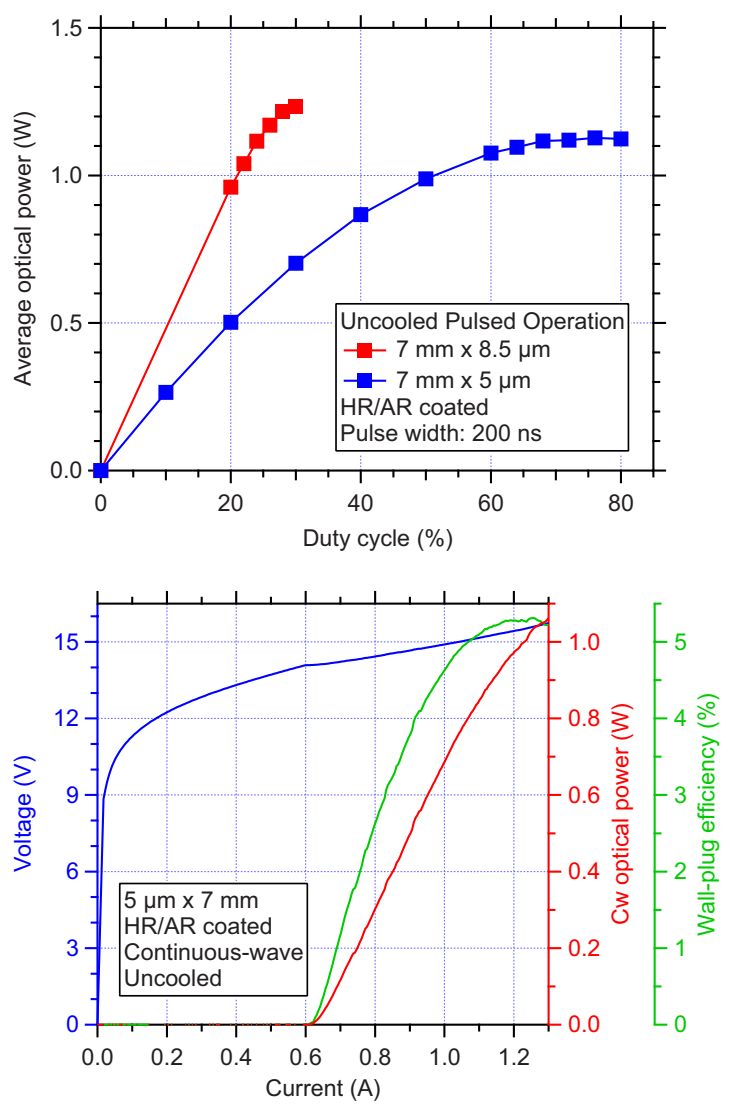

FIG. 3. (Color online) Top: average output power as a function of the duty cycle of uncooled 7-mm-long, HR/AR coated pulsed QCLs. Bottom: lightcurrent-voltage characteristic of an uncooled cw QCL.

to increase up to a duty cycle of $30 \%$, where it reached a maximum value of $1.23 \mathrm{~W}$. The average output power of the $5-\mu \mathrm{m}$-wide chip reached a maximum value of $1.13 \mathrm{~W}$ at a much higher duty cycle of $76 \%$. This chip was also operated in $\mathrm{cw}$ mode in the same configuration. Its measured cw output power, voltage, and WPE as functions of current are shown at the bottom part of Fig. 3. The maximum cw output power was $1.06 \mathrm{~W}$ and the maximum WPE was $5.3 \%$. These are, to date, the highest average and $\mathrm{cw}$ output powers reported for uncooled QCLs.

As mentioned earlier, nonuniform gain saturation in long devices reduces the WPE even with optimized reflectivity coatings. In this case, the WPE cannot be expressed by the simple formula shown in Eq. (1). One has to numerically solve the nonlinear differential equation for the longitudinal intensity profile. ${ }^{7,9}$ We did so using the numerical method described in Ref. 9. The cold cavity gain was estimated from the measurements described above: $G_{0}=g \Gamma\left(J_{\max }-J_{\text {tr }}\right)$ $=14.5 \mathrm{~cm}^{-1}$. As reported in Ref. 9, the optimum value for $\alpha_{m}$ is close to the value obtained through the use of the Rigrod model, but the WPE is reduced for long cavities. Relative WPE, i.e., normalized to the value obtained in the limit of short cavity lengths for which nonuniform gain saturation is negligible, versus cavity length computed in this framework is shown in Fig. 4. For the measured value of waveguide losses, $\alpha_{w}=0.5 \mathrm{~cm}^{-1}$, WPE is reduced by only $1.5 \%$ for $L=7 \mathrm{~mm}$ and by $4 \%$ for $L=10 \mathrm{~mm}$. Output power can thus still be further increased by using longer cavities without significant reduction in WPE. This is in contrast to diode lasers, whose $\alpha_{w}$ is typically $\sim 5 \mathrm{~cm}^{-1},{ }^{11}$ and whose WPE thus decreases very rapidly with increasing $L$, limiting the

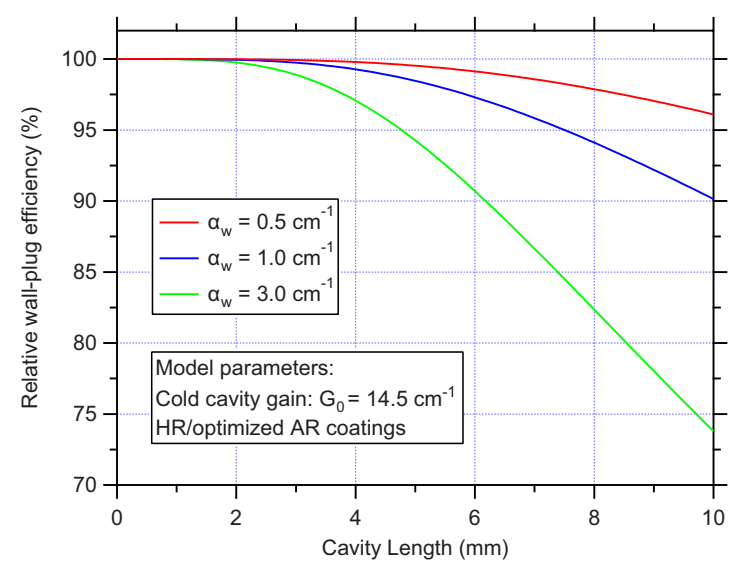

FIG. 4. (Color online) Dependence of WPE of chips with HR and optimized AR coatings on cavity length computed using Schindler's method.

size of most devices to a few millimeters or less. For higher waveguide losses of $3 \mathrm{~cm}^{-1}$, typical of the optimized longwave infrared QCLs, ${ }^{12}$ this effect becomes more significant. The WPE is reduced by $26 \%$ for $L=10 \mathrm{~mm}$.

In conclusion, we have demonstrated that the output power of QCLs can be increased while maintaining high WPE by using long cavities and optimized AR coatings. We reported $\mathrm{cw}$ output power of $2.9 \mathrm{~W}$ at room temperature for thermoelectrically cooled devices mounted on AlN submounts and average output power of $1.2 \mathrm{~W}$ and $\mathrm{cw}$ power of $1.1 \mathrm{~W}$ for uncooled devices. This record high uncooled performance has major consequences for applications of high power QCLs because it allows significant reduction in the power consumption of the laser system by removing the need for a TEC and, thus, facilitates integration into applications such as portable chemical sensors, free-space optical communications, and handheld MWIR illuminators for nightvision applications. Finally, we have shown theoretically that because of the low waveguide losses of these devices, output power can be further increased with negligible degradation of WPE by using cavity lengths up to $\sim 1 \mathrm{~cm}$ and appropriate AR coatings.

This work was supported in part through DARPA under Contract No. W911QX-07-C-0041 (approved for public release, Distribution Unlimited).

${ }^{1}$ Y. Bai, S. R. Darvish, S. Slivken, W. Zhang, A. Evans, J. Nguyen, and M. Razeghi, Appl. Phys. Lett. 92, 101105 (2008).

${ }^{2}$ A. Lyakh, C. Pflügl, L. Diehl, Q. J. Wang, F. Capasso, X. J. Wang, J. Y. Fan, T. Tanbun-Ek, R. Maulini, A. Tsekoun, R. Go, C. Kumar, and N. Patel, Appl. Phys. Lett. 92, 111110 (2008).

${ }^{3}$ Y. Bai, S. Slivken, S. R. Darvish, and M. Razeghi, Appl. Phys. Lett. 93, 021103 (2008).

${ }^{4}$ A. Lyakh, R. Maulini, A. Tsekoun, R. Go, C. Pflügl, L. Diehl, Q. J. Wang, F. Capasso, and C. Kumar N. Patel, Appl. Phys. Lett. 95, 141113 (2009).

${ }^{5}$ X. Liu, R. W. Davis, L. C. Hughes, M. H. Rasmussen, R. Bhat, C.-E. Zah, and J. Stradling, J. Appl. Phys. 100, 013104 (2006).

${ }^{6}$ A. Tsekoun, R. Go, M. Pushkarsky, M. Razeghi, and C. K. N. Patel, Proc. Natl. Acad. Sci. U.S.A. 103, 4831 (2006).

${ }^{7}$ W. W. Rigrod, IEEE J. Quantum Electron. 14, 377 (1978).

${ }^{8}$ J. S. Yu, S. Slivken, A. Evans, and M. Razeghi, IEEE J. Quantum Electron. 44, 747 (2008).

${ }^{9}$ G. M. Schindler, IEEE J. Quantum Electron. 16, 546 (1980).

${ }^{10}$ A. Wittmann, A. Hugi, E. Gini, N. Hoyler, and J. Faist, IEEE J. Quantum Electron. 44, 1083 (2008).

${ }^{11}$ L. A. Coldren and Scott W. Corzine, Diode Lasers and Photonics Integrated Circuits (Wiley, New York, 1995).

${ }^{12}$ A. Bismuto, T. Gresch, A. Bächle, and J. Faist, Appl. Phys. Lett. 93, 231104 (2008). 